\title{
AN APPLICATION OF THE O'NAN-SCOTT THEOREM TO THE GROUP GENERATED BY THE ROUND FUNCTIONS OF AN AES-LIKE CIPHER
}

\author{
A. CARANTI, F. DALLA VOLTA, AND M. SALA
}

\begin{abstract}
In a previous paper, we had proved that the permutation group generated by the round functions of an AES-like cipher is primitive. Here we apply the O'Nan Scott classification of primitive groups to prove that this group is the alternating group.
\end{abstract}

\section{INTRODUCTION}

According to Shannon [Sha49, p. 657], a cipher "is defined abstractly as a set of transformations". Coppersmith and Grossman [CG75], and later in 1988 Kaliski, Rivest and Sherman [KRS88, called attention to the group generated by a cipher. One of the motivations for the work of Kaliski et al. is that at that time Triple DES was being suggested as an improvement to DES. This meant replacing the use of single DES transformation $T_{a}$, where $a$ is a key, with the composition $T_{a} T_{b} T_{c}$, where $a, b, c$ are three DES keys. If it was the case that the transformations of DES form a group, then Triple DES would have been of course no more than DES itself. More generally, Kaliski et al. showed that if the group generated by the transformations of a cipher is too small, then the cipher is exposed to certain cryptanalytic attacks.

It was later proved by Wernsdorf Wer93 that the group generated by the round functions of DES (which are even permutations) is the alternating group. This implies that the group generated by the DES transformations with independent subkeys is also the alternating group. (We are not aware of any work in this context that tries to take account of the key schedule.)

Wernsdorf used ad hoc methods in WWer02 to prove that the permutation group $G$ generated by the round functions of AES is the alternating group. (Here, too, these functions are even permutations.) Sparr and Wernsdorf have recently given another, permutation group theoretic proof in SW08.

The goal of this paper is to give a different proof of this fact, building upon our earlier paper [CDVS08]. There we had proved that the group $G$ is primitive.

Date: 15 March 2009.

2000 Mathematics Subject Classification. Primary 94A60; secondary 20B15 20E22.

Key words and phrases. cryptosystems, Rijndael, AES, groups generated by round functions, primitive groups, O'Nan-Scott, wreath products, affine groups.

First author partially supported by MIUR-Italy via PRIN 2006014340-002 "Lie algebras and rings. Groups. Cryptography". Second author partially supported by MIUR-Italy via PRIN 2007 "Group theory and applications". 
In the course of doing that we answered a question of Paterson [Pat99] about the possibility of embedding a trapdoor in a cipher by having the group generated by the cipher act imprimitively.

In this paper we work under certain cryptographic assumptions (see Section 2) that are a stripped down, simplified version of those of [CDVS08. (These are also satisfied by AES.) We first give, for the convenience of the reader, a short group-theoretic version of the main result of [CDVS08] under these assumptions. We then appeal to the O'Nan-Scott classification of primitive groups to prove that the group generated by the round functions of a cryptosystem satisfying our assumptions is the alternating group.

We are very grateful to Ralph Wernsdorf for several useful suggestions.

\section{Preliminaries}

In the rest of the paper, we tend to adopt the notation of [DR02].

Let $V=V(d, 2)$, the vector space of dimension $d$ over the field $\mathrm{GF}(2)$ with two elements, be the state (or message) space. $V$ has $n=2^{d}$ elements.

For any $v \in V$, consider the translation by $v$, that is the map

$$
\begin{aligned}
\sigma_{v}: V & \rightarrow V, \\
w & \mapsto w+v .
\end{aligned}
$$

In particular, $\sigma_{0}$ is the identity map on $V$. The set

$$
T=\left\{\sigma_{v}: v \in V\right\}
$$

is an elementary abelian, regular subgroup of $\operatorname{Sym}(V)$. In fact, the map

$$
\begin{aligned}
V & \rightarrow T \\
v & \mapsto \sigma_{v}
\end{aligned}
$$

is an isomorphism of the additive group $V$ onto the multiplicative group $T$.

We consider a key-alternating block cipher (see Section 2.4.2 of [DR02]) which consists of a fixed number of iterations of a function of the form $\rho \sigma_{k}$, where $k \in V$. Such a function is called a round function, and the parameter $k$ is called the round key. (We write maps left-to-right, so $\rho$ operates first.) Here $\rho$ is a fixed permutation operating on the vector space $V$. Therefore each round consists of an application of $\rho$, followed by a key addition. This covers for instance AES with independent subkeys. Let $G=\left\langle\rho \sigma_{k}: k \in V\right\rangle$ be the group of permutations of $V$ generated by the round functions. Choosing $k=0$ we see that $\rho \in G$, and thus $T \leq G$. It follows that $G=\langle T, \rho\rangle$.

We assume $\rho=\gamma \lambda$, where $\gamma$ and $\lambda$ are permutations. Here $\gamma$ is a bricklayer transformation, consisting of a number of S-boxes. The message space $V$ is written as a direct sum

$$
V=V_{1} \oplus \cdots \oplus V_{n_{t}}
$$

$n_{t}>1$, where each $V_{i}$ has the same dimension $m>1$ over $\operatorname{GF}(2)$. As $n_{t}>1$, this implies that $d=m n_{t}$ is not a prime number. For $v \in V$, we will write 
$v=v_{1}+\cdots+v_{n_{t}}$, where $v_{i} \in V_{i}$. Also, we consider the projections $\pi_{i}: V \rightarrow V_{i}$, which map $v \mapsto v_{i}$. We have

$$
v \gamma=v_{1} \gamma_{1} \oplus \cdots \oplus v_{n_{t}} \gamma_{n_{t}}
$$

where the $\gamma_{i}$ are S-boxes, which we allow to be different for each $V_{i}$.

$\lambda$ is a linear function (usually called a linear mixing layer). The only assumption we will be making about $\lambda$ is Cryptographic Assumption (3) below.

In AES the S-boxes are all equal, and consist of inversion in the field $\operatorname{GF}\left(2^{8}\right)$ with $2^{8}$ elements (see later in this paragraph), followed by an affine transformation, that is, a linear transformation, followed by a translation. When interpreting AES in our scheme, we take advantage of the well-known possibility of moving the linear part of the affine transformation to the linear mixing layer, and incorporating the translation in the key addition (see for instance [MR02]). Thus in our scheme for AES we have $m=8$, we identify each $V_{i}$ with $\operatorname{GF}\left(2^{8}\right)$, and we take $x \gamma_{i}=x^{2^{8}-2}$, so that $\gamma_{i}$ maps nonzero elements to their inverses, and zero to zero. As usual we will simply say that $\gamma_{i}$ acts by inversion.

We will work under the following

Cryptographic Assumptions. Consider an AES-like cryptosystem as described above, which satisfies the following conditions.

(1) $0 \gamma=0$ and $\gamma^{2}=1$, the identity transformation.

(2) There is $1 \leq r<m / 2$ such that the following hold.

(a) For all $0 \neq v \in V_{i}$, the image of the map $V_{i} \rightarrow V_{i}$, which maps $x \mapsto(x+v) \gamma_{i}+x \gamma_{i}$, has size greater than $2^{m-r-1}$, and it is not a coset of a subspace.

(b) There is no subspace of $V_{i}$, invariant under $\gamma_{i}$, of codimension less than or equal to $2 r$.

(3) There are no subspaces $U, U^{\prime}, U^{\prime \prime}$ (except $\{0\}$ and $V$ ) that are the sum of some of the $V_{i}$, and such that $U \lambda=U^{\prime}$ and $U^{\prime} \lambda=U^{\prime \prime}$.

In CDVS08 we have proved under certain abstract and general assumptions a result that specializes to the following:

Theorem 1. Suppose a cryptosystem satisfies the Cryptographic Assumptions. Then the group $G$ generated by its round functions acts primitively on the message space $V$.

We give a short, group-theoretic proof of this in Section 3 . This we do for the convenience of the reader, as we will need to refer to part of the proof in Section 6 . We are grateful to the referee of another paper for this proof.

In the rest of the paper we prove the following

Theorem 2. Suppose a cryptosystem satisfies the Cryptographic Assumptions.

Then the group $G$ generated by its round functions is the alternating group $\operatorname{Alt}(V)$.

The same holds for the group generated by the cryptosystem with independent subkeys. 
A word about the parity of the group $G$ is in order here. Over $V=V(d, 2)$, non-trivial translations are clearly involutions without fixed points, and thus even permutations. Also, for $d>2$ the group $\operatorname{GL}(d, 2)=\operatorname{SL}(d, 2)$ is perfect, so that in particular it has no (normal) subgroup of order 2 , and it is thus contained in $\operatorname{Alt}(V)$.

We now show that $\gamma$ is also even, so that $G \leq \operatorname{Alt}(V)$. In fact, $\gamma$ is the product of $n_{t}$ permutations $g_{i}$, acting as $\gamma_{i}$ on $V_{i}$, and as the identity on $V_{j}, j \neq i$. This means that every 2-cycle in $\gamma_{i}$ gives rise to $2^{d-m} 2$-cycles in $g_{i}$. Now the number $2^{d-m}$ is even, as $d-m=n_{t} m-m>m, n_{t}>1$ by assumption, and $m>2$ by Cryptographic Assumption (11). It follows that each $g_{i}$ is even, and thus so is $\gamma$. (The same argument proves that $\gamma$ is even, even without assuming that it is an involution, as we do here.)

Condition (1) is clearly satisfied by AES. As we said above, we take advantage here of the possibility of assuming that $\gamma$ is simply componentwise inversion.

Condition (2a) is also well-known to be satisfied, with $r=1$ (see Nyb94 but also [DR06]), as the image of that map has size $2^{7}-1$.

As to Condition (2b), it is also satisfied by AES with $r=1$. For that, one could just use GAP GAP05 to verify that the only nonzero subspaces of $\mathrm{GF}\left(2^{8}\right)$ which are invariant under inversion are the subfields. However, this can also be derived from a more general result of GGSZ06] and [Mat07], which states that the only nonzero additive subgroups of $\mathrm{GF}\left(2^{m}\right)$, which contain the inverse of all of their nonzero elements, are the subfields.

Condition (3) follows from the properties of the components MixColumns [DR02, 3.4.3] and ShiftRows [DR02, 3.4.2] of the linear mixing layer (which are not altered by the fact that we have incorporated in it the linear part of the S-boxes). In fact, suppose, without loss of generality, that $U \supseteq V_{1}$. Then $U^{\prime}$ contains the whole first column of the state, and $U^{\prime \prime}=V$, a contradiction. This argument is a vestigial form of the Four-Round Propagation Theorem [DR02, 9.5.1].

\section{Primitivity}

In this section we give a proof of Theorem 1.

Suppose for a contradiction that $G=\langle T, \rho\rangle$ is imprimitive on $V$, so that any block system for $G$ is given by the cosets of some subspace $U$ of $V$. This is because, as it is proved in CDVS08, a block system for $G$ is also a block system for the group $T$ of translations.

Now $\rho=\gamma \lambda$, with $\lambda$ linear, and $0 \gamma=0$. Thus $U \rho=U$, and $U^{\prime}=U \gamma=U \lambda^{-1}$ is a subspace.

Suppose firstly that $U=V_{i_{1}} \oplus \cdots \oplus V_{i_{l}}$ is a direct sum of some of the subspaces $V_{i}\left(l<n_{t}\right)$. Then, $U^{\prime}=U \gamma=U$, so that $U^{\prime}=U$ is $\lambda$-invariant; this contradicts Cryptographic Assumption (3).

Thus there exists $i$ such that $U \nsupseteq V_{i}$, but there is $u \in U$, such that its $i$ th component $u_{i} \in V_{i}$ is nonzero. We claim that $U \cap V_{i}$ is nonzero. Take any $v \in V_{i}$. Then $(u+v) \gamma+v \gamma \in U^{\prime}$, so that $u \gamma+(u+v) \gamma+v \gamma \in U^{\prime}$. The latter element has all zero components, expect possibly the $i$-th one, which is $u_{i} \gamma_{i}+\left(u_{i}+v\right) \gamma_{i}+v \in U^{\prime} \cap V_{i}$. Were the latter zero for all $v \in V_{i}$, then the map 
$V_{i} \rightarrow V_{i}$ that maps $v \mapsto\left(u_{i}+v\right) \gamma_{i}+v \gamma_{i}$ would be constant, thus contradicting Cryptographic Assumption (2a).

Thus there exists $i$ such that both $U_{i}=U \cap V_{i}$ and $U_{i}^{\prime}=\left(U_{i}\right) \gamma_{i}=U^{\prime} \cap V_{i}$ are nonzero, proper subspaces of $V_{i}$ of the same dimension, and

$$
\gamma_{i}: V_{i} / U_{i} \rightarrow V_{i} / U_{i}^{\prime}
$$

If $x \in V_{i}$, and $v \in U_{i}, v \neq 0$, then $x+v$ and $x$ are in the same coset of $U_{i}$, so $(x+v) \gamma_{i}$ and $x \gamma_{i}$ are in the same coset of $U_{i}^{\prime}$. Thus the set

$$
\left\{(x+v) \gamma_{i}+x \gamma_{i}: x \in V_{i}\right\}
$$

is a subset of $U_{i}^{\prime}$, and by Cryptographic Assumption (2a) $U_{i}$ and $U_{i}^{\prime}$ have size greater than $2^{m-r-1}$, that is to say dimension at least $m-r$ or equivalently codimension at most $r$. The codimension of $U_{i} \cap U_{i}^{\prime}$ is therefore at most $2 r$, so $U_{i} \cap U_{i}^{\prime}$ cannot be $\gamma_{i}$-invariant because of Cryptographic Assumption (2b). This means there exists $z \in U_{i} \cap U_{i}^{\prime}$ such that $z \gamma_{i} \notin U_{i} \cap U_{i}^{\prime}$, so $z \gamma_{i} \notin U_{i}$, as $z \gamma_{i} \in U_{i}^{\prime}$. However, $U_{i}^{\prime}$ is the image of $U_{i}$ under the bijective map $\gamma_{i}$, so $z=z \gamma_{i}^{2} \notin U_{i}^{\prime}$, as $z \gamma_{i} \notin U_{i}$. Thus $z \notin U_{i} \cap U_{i}^{\prime}$, which is a contradiction.

\section{O'NAN-SCOTT}

In this section we prove Theorem 2, We first state the O'Nan-Scott classification of primitive groups for the case of the maximal primitive subgroups of the symmetric group. We give the result for the symmetric group of degree $q^{n}$, where $q$ is a power of a prime number $p$.

Theorem 3. [Cam99, Theorem 4.8] Suppose $q$ is a power of the prime $p$.

A maximal primitive subgroup $G$ of $\operatorname{Sym}\left(q^{n}\right)$ is one of the following:

(1) affine, that is, $G=\operatorname{AGL}(d, p), p^{d}=q^{n}$, for some $d$;

(2) primitive non-basic, that is, a wreath product $G=\operatorname{Sym}(k)<\operatorname{Sym}(r)$ in product action, $k^{r}=q^{n}, k \neq 2, r>1$.

(3) almost simple, that is, $S \leq G \leq \operatorname{Aut}(S)$, for a nonabelian simple group $S$.

Note that in our context $p=2$.

It is convenient to use a refinement of the O'Nan-Scott theorem, due to Cai Heng Li [Li03], for the special case when $G$ contains an abelian regular subgroup $T$; in our case, this is the group of translations.

Theorem 4. [Li03, Theorem 1.1] Let $G$ be a primitive group of degree $2^{d}$, with $d \geq 1$. Suppose $G$ contains a regular abelian subgroup $T$.

Then $G$ is one of the following

(1) affine, that is, $G \leq \operatorname{AGL}(d, 2)$;

$$
G=\left(S_{1} \times \cdots \times S_{r}\right) . O . P,
$$

with $2^{d}=m^{r}$ for some $m$ and $r>1$. Here $T=T_{1} \times \cdots \times T_{r}$, with $T_{i}<S_{i} \cong \operatorname{Alt}(m)$ for each $i, O \leq \operatorname{Out}\left(S_{1}\right) \times \cdots \times \operatorname{Out}\left(S_{r}\right)$, and $P$ permutes transitively the $S_{i}$.

(3) almost simple, that is, $S \leq G \leq \operatorname{Aut}(S)$, for a nonabelian simple group $S$. 
To prove the first statement of Theorem 2 we need to deal with the three possible cases of Theorem 4.

Case (1) is treated in Section 5, An important observation of Li [Li03] is in order here. If $V$ is a vector space, with addition + , then the symmetric group $\operatorname{Sym}(V)$ contains the affine group $\operatorname{AGL}(V)=T \mathrm{GL}(V)$, where $T$ is the group of translations. But $\operatorname{Sym}(V)$ also contains the conjugates of $\operatorname{AGL}(V)$, which are still affine groups on the set $V$, but possibly with respect to an operation $\circ$ different from + . In particular the group $T$ of translations may be contained in one of these conjugates, where it will be an abelian regular subgroup. We have studied this situation in CDVS06, and we will be exploiting these results in Section 5.

Case (2) will be dealt with in Section 6.

In the almost simple case (3), the intersection of a one-point stabilizer in $G$ with $S$ is a proper subgroup of $S$ of index $2^{d}$, since the nontrivial normal subgroup $S$ of the primitive group $G$ is transitive. We can thus appeal (as Li does) to a particular case of a result of Guralnick Gur83], which states that the only nonabelian simple groups that have a subgroup of index of the form $2^{d}$ are either the alternating groups $S=\operatorname{Alt}\left(2^{d}\right)$, with $d>2$, or the groups $\operatorname{PSL}(f, q)$, where $q$ is a primepower, and $f$ is prime, $\left(q^{f}-1\right) /(q-1)=2^{d}$. We rule out the second possibility as follows. Since $\left(q^{f}-1\right) /(q-1)=q^{f-1}+q^{f-2}+\cdots+q+1 \equiv f(\bmod 2)$, we have $f=2$ here, and $q=2^{d}-1$. Well-known elementary arguments yield that $q$ and $d$ are prime. However, $d=n_{t} m$ is not prime, as $n_{t}>1$ by assumption, and as noted earlier $m>2$ by Cryptographic Assumption (11).

Clearly $\operatorname{Aut}\left(\operatorname{Alt}\left(2^{d}\right)\right)=\operatorname{Sym}\left(2^{d}\right)$ here, so $G$ is either the alternating or the symmetric group. Since we have shown in Section 2 that $G \leq \operatorname{Alt}(V)$, we obtain $G=\operatorname{Alt}(V)$.

To prove the second statement of Theorem 2, we then appeal to a standard argument: if the nonabelian simple group $G$ is generated by a subset $S$, then for any fixed $r$ the set $S^{\prime}=\left\{s_{1} s_{2} \ldots s_{r}: s_{i} \in S\right\}$ of $r$-fold products of elements of $S$ generates a nontrivial normal subgroup of $G$, and thus $S^{\prime}$ also generates $G$. In our context $S$ is the set of the round functions for all possible subkeys, and $r$ is the number of rounds, so that $S^{\prime}$ is the set of the transformations of the cryptosystem with independent subkeys.

\section{THE AFFine CASE}

Suppose $G$ is contained in an affine subgroup of $\operatorname{Sym}(V)$. By the theory of CDVS06, there is a structure of an associative, commutative, nilpotent ring $(V, \circ, \cdot, 0)$ on $V$, such that $(V, \circ, 0)$ is a vector space over the field with two elements, and ordinary addition on $V$ is expressed as

$$
x+y=x \circ y \circ x y,
$$

for $x, y \in V$. Moreover, $G$ acts as a group of affine transformations on $(V, \circ, 0)$.

As both $(V, \circ, 0)$ and $(V,+, 0)$ are elementary abelian, we have

$$
0=x+x=x \circ x \circ x x=0 \circ x^{2}=x^{2}
$$


for all $x \in V$. It follows

$$
\begin{aligned}
x+y+x y & =(x \circ y \circ x y) \circ x y \circ(x \circ y \circ x y) \cdot x y \\
& =x \circ y \circ x y \circ x y \circ x^{2} y \circ x y^{2} \circ x^{2} y^{2} \\
& =x \circ y .
\end{aligned}
$$

Here we have used the fact that - distributes over $\circ$.

Now $\rho \in G$ is linear with respect to $\circ$, that is $(x \circ y) \rho=x \rho \circ y \rho$ for all $x, y \in V$. Choose $0 \neq y \in U=\{z \in V: x z=0$ for all $x \in V\}$. (The latter set is different from $\{0\}$, as the ring $(V, \circ, \cdot, 0)$ is nilpotent.) Then

$$
(x+y) \rho=(x \circ y) \rho=x \rho \circ y \rho=x \rho+y \rho+x \rho \cdot y \rho .
$$

Now note that given $x \in V$, the set $x V=\{x z: z \in V\}$ is a subspace with respect to $\circ$, as - distributes over $\circ$; and also a subspace with respect to + , as $x z_{1}+x z_{2}=x z_{1} \circ x z_{2} \circ x^{2} z_{1} z_{2}=x z_{1} \circ x z_{2}$.

It follows from 5.1 that for $0 \neq y \in U$ we have

$$
\{(x+y) \rho+x \rho: x \in V\}=y \rho+y \rho V .
$$

The right hand side is a coset of a subspace of $V$ with respect to + . Now $\lambda$ (and its inverse) are linear with respect to + . Applying $\lambda^{-1}$ we obtain that

$$
\{(x+y) \gamma+x \gamma: x \in V\}
$$

is also a coset of a subspace of $V$ with respect to + . Choose an index $i$ so that the component $y_{i} \in V_{i}$ of $y$ is nonzero. Then we have that the projection on $V_{i}$ of the previous set

$$
\left\{\left(x+y_{i}\right) \gamma+x \gamma: x \in V_{i}\right\}
$$

is a coset of a subspace of $V_{i}$ with respect to + . This contradicts Cryptographic Assumption (2a).

\section{WREATH PRODUCT IN PRODUCT ACTION}

Here we deal to the case when

$$
G=\left(S_{1} \times \cdots \times S_{r}\right) . O . P,
$$

with $2^{d}=k^{r}$ for some $k$ and $r>1$. Here $T=T_{1} \times \cdots \times T_{r}$, where $\left|T_{i}\right|=k$ and $T_{i}<S_{i} \cong \operatorname{Alt}(k)$ for each $i, O \leq \operatorname{Out}\left(S_{1}\right) \times \cdots \times \operatorname{Out}\left(S_{r}\right)$, and $P$ permutes transitively the $S_{i}$ by conjugation. It follows that $S_{1} \times \cdots \times S_{r}=\operatorname{Soc}(G)$.

Note that if $k=2$ or 4 , so that $S_{i} \cong \operatorname{Alt}(2)$ or $\operatorname{Alt}(4)$, the group $T$ of translations is normal in $G$, so that $G \leq \operatorname{AGL}(V)$. This contradicts the non-linearity of $\gamma$, which follows from Cryptographic Assumption (2a). Thus we will assume $k>4$ in the rest of this section.

Note that $G=\langle T, \rho\rangle$, and $T \leq \operatorname{Soc}(G)$, so that $G / \operatorname{Soc}(G)$ is cyclic, spanned by $\rho$. Since $P$ permutes transitively the $S_{i}$, it follows that $\rho$ permutes cyclically the $S_{i}$ by conjugation, that is, we may rename indices so that $S_{i}^{\rho}=\rho^{-1} S_{i} \rho=S_{i+1}$ for each $i$ (and indices are taken modulo $r$ ). 
Since each $T_{i}$ is a group of translations, $W_{i}=0 T_{i} \subseteq 0 S_{i}$ is a subspace of $V$, of order $k$. Since $0 S_{i}$ has also order $k, 0 T_{i}=0 S_{i}$. Clearly each element of $v \in V$ can be written uniquely in the form $v=0 t$, for $t \in T$. Thus

$$
v=0 t_{1} t_{2} \ldots t_{r}=0 t_{1}+0 t_{2}+\cdots+0 t_{r}
$$

for unique $t_{i} \in T_{i}$, and

$$
V=W_{1} \oplus W_{2} \oplus \cdots \oplus W_{r}
$$

For each $i$ we have also $W_{i} \rho=0 S_{i} \rho=0 S_{i+1}^{\rho^{-1}} \rho=0 \rho S_{i+1}=0 S_{i+1}=W_{i+1}$, as $0 \rho=0$. Thus $\rho$ permutes cyclically the $W_{i}$. Now let $v \in V$, and write it as $v=w_{1}+\cdots+w_{r}$ where $w_{i} \in W_{i}$. Let $t_{i} \in W_{i}$ be such that $w_{i}=0 t_{i}$. Since the $t_{i}$ are translations, we have $v=0 t_{1}+0 t_{2}+\cdots+0 t_{r}=0 t_{1} t_{2} \ldots t_{r}$. We have $v \rho=0 t_{1} t_{2} \ldots t_{r} \rho=0 t_{1}^{\rho} t_{2}^{\rho} \ldots t_{r}^{\rho}$, as $0 \rho^{-1}=0$. Since $t_{i}^{\rho} \in S_{i}^{\rho}=S_{i+1}$, there are $t_{i}^{\prime} \in T_{i}$ such that $0 t_{i}^{\rho}=0 t_{i} \rho=0 t_{i+1}^{\prime} \in W_{i+1}$, and because $S_{i}$ and $S_{j}$ commute elementwise, we have

$$
\begin{aligned}
v \rho & =0 t_{1}^{\rho} t_{2}^{\rho} \ldots t_{r}^{\rho}=0 t_{2}^{\prime} t_{2}^{\rho} \ldots t_{r}^{\rho}=0 t_{2}^{\rho} t_{2}^{\prime} \ldots t_{r}^{\rho} \\
& =0 t_{3}^{\prime} t_{2}^{\prime} \ldots t_{r}^{\rho}=0 t_{2}^{\prime} t_{3}^{\prime} \ldots t_{r}^{\rho}=\ldots \\
& =0 t_{2}^{\prime} t_{3}^{\prime} \ldots t_{1}^{\prime}=0 t_{1}^{\prime}+0 t_{2}^{\prime}+\cdots+0 t_{r}^{\prime} \\
& =0 t_{r} \rho+0 t_{1} \rho+\cdots+0 t_{r-1} \rho \\
& =w_{1} \rho+w_{2} \rho+\cdots+w_{r} \rho .
\end{aligned}
$$

Now fix an index $i$, and take $u \in W_{i}$. We have from the above

$$
v \rho=\left(w_{1}+w_{2}+\cdots+w_{r}\right) \rho=w_{1} \rho+w_{2} \rho++\cdots+w_{r} \rho,
$$

where $w_{i} \rho \in W_{i+1}$, and also

$$
(v+u) \rho=w_{1} \rho+\left(w_{i}+u\right) \rho+\cdots+w_{r} \rho
$$

with $\left(w_{i}+u\right) \rho \in W_{i+1}$. It follows

$$
(v+u) \rho+v \rho=w_{i} \rho+\left(w_{i}+u\right) \rho \in W_{i+1} .
$$

Now $\rho=\gamma \lambda$, where $\lambda$ is linear. Applying $\lambda^{-1}$ to both sides of (6.1) we get $(v+u) \gamma+v \gamma \in W_{i+1} \lambda^{-1}$. In other words, there are subspaces $W_{i}, W_{i+1} \lambda^{-1}$ of $V$ of the same dimension such that when the input difference to $\gamma$ is in the first one, then the output difference is in second one. By the arguments of Section 3 (with $U=W_{i}$ and $\left.U^{\prime}=W_{i+1} \lambda^{-1}\right)$, it follows that $W_{i}$ is the direct sum of some of the $V_{j}$, for each $i$. Thus $W_{2}=W_{1} \rho=W_{1} \lambda$ and $W_{3}=W_{2} \lambda$, contradicting Cryptographic Assumption (3).

\section{REFERENCES}

[Cam99] Peter J. Cameron, Permutation groups, London Mathematical Society Student Texts, vol. 45, Cambridge University Press, Cambridge, 1999. MR 2001c:20008

[CDVS06] A. Caranti, F. Dalla Volta, and M. Sala, Abelian regular subgroups of the affine group and radical rings, Publ. Math. Debrecen 69 (2006), no. 3, 297-308. MR MR2273982 (2007j:20001)

[CDVS08] A Caranti, F. Dalla Volta, and M. Sala, On some block ciphers and imprimitive groups, http://arxiv.org/abs/math/0806.4135, 2008. 
[CG75] Don Coppersmith and Edna Grossman, Generators for certain alternating groups with applications to cryptography, SIAM J. Appl. Math. 29 (1975), no. 4, 624-627. MR MR0495175 (58 \#13909)

[DR02] Joan Daemen and Vincent Rijmen, The design of Rijndael, Information Security and Cryptography, Springer-Verlag, Berlin, 2002, AES - the advanced encryption standard. MR MR1986943 (2006b:94025)

[DR06] Joan Daemen and Vincent Rijmen, Two-round AES differentials, IACR e-print eprint.iacr.org/2006/039.pdf, 2006.

[GAP05] The GAP Group, GAP - Groups, Algorithms, and Programming, Version 4.4, 2005, ( \protect \vrule width0pt \protect \href\{http://www.gap-system.org\}\{http://www.gap-syst

[GGSZ06] Daniel Goldstein, Robert M. Guralnick, Lance Small, and Efim Zelmanov, Inversion invariant additive subgroups of division rings, Pacific J. Math. 227 (2006), no. 2, 287-294. MR MR2263018 (2007i:17041)

[Gur83] Robert M. Guralnick, Subgroups of prime power index in a simple group, J. Algebra 81 (1983), no. 2, 304-311. MR 84m:20007

[KRS88] Burton S. Kaliski, Jr., Ronald L. Rivest, and Alan T. Sherman, Is the data encryption standard a group? (Results of cycling experiments on DES), J. Cryptology 1 (1988), no. 1, 3-36. MR MR935899 (89f:94017)

[Li03] Cai Heng Li, The finite primitive permutation groups containing an abelian regular subgroup, Proc. London Math. Soc. (3) 87 (2003), no. 3, 725-747. MR MR2005881 (2004i:20003)

[Mat07] Sandro Mattarei, Inverse-closed additive subgroups of fields, Israel J. Math. 159 (2007), 343-347. MR MR2342485

[MR02] Sean Murphy and Matthew J. B. Robshaw, Essential algebraic structure within the AES, Advances in cryptology - CRYPTO 2002, Lecture Notes in Comput. Sci., vol. 2442, Springer, Berlin, 2002, pp. 1-16. MR MR2054809 (2005a:94064)

[Nyb94] Kaisa Nyberg, Differentially uniform mappings for cryptography, Advances in cryptology_EUROCRYPT '93 (Lofthus, 1993), Lecture Notes in Comput. Sci., vol. 765, Springer, Berlin, 1994, pp. 55-64. MR MR1290329 (95e:94039)

[Pat99] Kenneth G. Paterson, Imprimitive permutation groups and trapdoors in iterated block ciphers, Fast Software Encryption: 6th International Workshop, FSE'99, Rome (L. Knudsen, ed.), Lecture Notes in Computer Science, vol. 1636, Springer-Verlag, Heidelberg, March 1999, pp. 201-214.

[Sha49] C. E. Shannon, Communication theory of secrecy systems, Bell System Tech. J. 28 (1949), 656-715. MR MR0032133 (11,258d)

[SW08] Rüdiger Sparr and Ralph Wernsdorf, Group theoretic properties of RIJNDAELlike ciphers, Discrete Appl. Math. 156 (2008), no. 16, 3139-3149, doi:10.1016/j.dam.2007.12.011.

[Wer93] Ralph Wernsdorf, The one-round functions of the DES generate the alternating group, Advances in cryptology-EUROCRYPT '92 (Balatonfüred, 1992), Lecture Notes in Comput. Sci., vol. 658, Springer, Berlin, 1993, pp. 99-112. MR MR1243663 (94g:94031)

[Wer02] Ralph Wernsdorf, The round functions of RIJNDAEL generate the alternating group, Proceedings of the 9th International Workshop on Fast Software Encryption, Lecture Notes in Computer Science, vol. 2365, Springer-Verlag, Heidelberg, 2002, FSE2002, Leuven, Belgium, February 2002, pp. 143-148. 
(A. Caranti) Dipartimento di Matematica, Università degli Studi di Trento, via Sommarive 14, I-38050 Povo (Trento), Italy

E-mail address: andrea.caranti@unitn.it

$U R L:$ http://science.unitn.it/ ${ }^{\sim}$ caranti/

(F. Dalla Volta) Dipartimento di Matematica e Applicazioni, Edificio U5, Università degli Studi di Milano-Bicocca, via Roberto Cozzi 53, I-20125 Milano, Italy

E-mail address: francesca.dallavolta@unimib.it

$U R L:$ http://www.matapp. unimib.it/ 〜allavolta/

(M. Sala) Dipartimento di Matematica, Università degli Studi di Trento, via Sommarive 14, I-38050 Povo (Trento), Italy

E-mail address: sala@science.unitn.it 